\title{
SEIFERT FIBERED SPACES IN IRREDUCIBLE, SUFFICIENTLY-LARGE 3-MANIFOLDS
}

\author{
BY WILLIAM JACO ${ }^{1}$ AND PETER B. SHALEN ${ }^{1}$
}

Communicated by P. J. Church, May 11, 1976

In [2], F. Waldhausen announced theorems about singular annuli and tori in a bounded, orientable, irreducible 3-manifold $M$, analogous to the Dehn LemmaLoop Theorem for singular disks and the Sphere Theorem for singular spheres. The Torus Theorem was used to prove that the centralizer of an element of $\pi_{1}(M)$ is finitely generated; as a corollary, this yields a simple proof of the earlier result that $\pi_{1}(M)$ has no infinitely divisible elements.

As consequences of the main theorem announced below, we obtain greatly sharpened versions of all the results mentioned above. We also obtain a canonical way of decomposing a rather general compact 3-manifold into submanifolds with nice properties.

Our main theorem is a homotopy-classification theorem for certain maps of Seifert fibered spaces and $I$-bundles into the 3-manifold $M$. An immediate consequence of the theorem, in effect a special case, is a homotopy-classification of singular annuli and tori in $M$. Similar results have been obtained independently by Johannson [1].

In what follows, manifolds are understood to be piecewise-linear. A Seifert fibered space or an $I$-bundle over a surface is understood to have a fixed fibration.

Definitions. A 3-manifold pair is a pair $(M, T)$, where $M$ is a 3-manifold and $T \subset \partial M$ is a 2 -manifold. The pair $(M, T)$ is sufficiently-large if $M$ is compact, connected, orientable, irreducible and sufficiently large while $T$ is compact and each component of $T$ is incompressible. A Seifert pair is a 3-manifold pair ( $S$, $F$ ), in which both $S$ and $F$ are compact and orientable, and such that for each component $S$ of $S$, there exists either (i) a homeomorphism of $S$ onto a Seifert fibered space, which maps $S \cap F$ onto a union of fibers, or (ii) a homeomorphism of $S$ onto a PL $I$-bundle over a surface, which maps $S \cap F$ onto the associated $\partial I$-bundle. The Seifert pair $(S, F)$, with $S$ connected, is called degenerate if either (i) $\pi_{1}(S)=\{1\}$, or (ii) $F=\varnothing$ and $\pi_{1}(S)$ is cyclic.

Let $(S, F)$ be a polyhedral pair such that $S$ is connected. A map of pairs

AMS (MOS) subject classifications (1970). Primary 57A10; Secondary 55A10, 55 A35. Key words and phrases. Seifert fibered space, centralizers, roots, Dehn Lemma, Loop Theorem, Sphere Theorem.

1 The research of both authors is partially supported by NSF Grant MPS 71-03022. 
$f:(S, F) \rightarrow(M, T)$, where $(M, T)$ is a 3-manifold pair, is essential if (i) $f_{*}: \pi_{1}(S)$ $\longrightarrow \pi_{1}(M)$ is a monomorphism, and (ii) $f$ cannot be homotoped as a map of pairs to a map $f^{\prime}$ such that $f^{\prime}(S) \subset T$.

MaIn TheOREM. Let $(M, T)$ be a sufficiently-large 3-manifold pair. Then there exists a Seifert pair $(\Sigma, \Phi)$, where $\Sigma \subset M, \Phi \subset T$, such that for any nondegenerate, connected Seifert pair $(S, F)$ and any essential map $f:(S, F) \rightarrow$ $(M, T), f$ is homotopic as a map of pairs to a map $g$ such that $g(S) \subset \Sigma$ and $g(F) \subset \Phi$.

It is immediate from this theorem that any essential map of $\left(S^{1} \times I, S^{1} \times\right.$ $\partial I)$ or $\left(S^{1} \times S^{1}, \varnothing\right)$ into $(M, T)$ is homotopic (as a map of pairs) to a map into $(\Sigma, \Phi)$. This version of the Torus-annulus Theorem may be used to prove Waldhausen's Theorem with no great difficulty.

THEOREM. Let $M$ be a compact, connected, orientable, irreducible and sufficiently large 3-manifold. Then there exists a collection (possibly empty) of disjoint, compact Seifert fibered spaces $\Sigma_{1}, \ldots, \Sigma_{k} \subset M$ with incompressible boundaries, and subgroups (well defined up to conjugacy) $G_{i}$ of index $\leqslant 2$ in $\bar{G}_{i}$ $\operatorname{Im}\left(\pi_{1}\left(\Sigma_{i}\right) \rightarrow \pi_{1}(M)\right)$, such that: (i) each $G_{i}$ is the centralizer of some element of $\pi_{1}(M)$, but no $G_{i}$ is cyclic; (ii) any noncyclic subgroup of $\pi_{1}(M)$ which is the centralizer of an element of $\pi_{1}(M)$ is conjugate in $\pi_{1}(M)$ to a subgroup of one of the groups $\bar{G}_{i}$; (iii) any subgroup of $\pi_{1}(M)$ which is the centralizer of an element of $\pi_{1}(M)$, and has no abelian subgroup of index $\leqslant 2$, is conjugate in $\pi_{1}(M)$ to one of the $G_{i}$.

The set of all roots of an element of the fundamental group of a Seifert fibered 3-manifold is easily described; and by combining this description with the above theorem on centralizers, one may obtain a description of the set of roots of any $x \in \pi_{1}(M)$ when $M$ is sufficiently large. In the case of a knot group, this answers a question of L. P. Neuwirth, and it is in this case that we shall state the result.

THEOREM. Let $K$ be a polyhedral knot in $S^{3}$ and let $M$ denote its associated knot space. Then there exists a collection (possibly empty) of disjoint torus knot spaces ${ }^{2} T_{1}, \ldots, T_{k} \subset M$ with incompressible boundaries, and having the following properties. (i) Any element of $\pi_{1}(M)$, the roots of which do not lie in a single subgroup of $\pi_{1}(M)$, is conjugate to an element of one of the subgroups (well defined up to conjugacy) $G_{i}=\operatorname{Im}\left(\pi_{1}\left(T_{i}\right) \rightarrow \pi_{1}(M)\right)$; (ii) the roots of any element of $G_{i}(1 \leqslant i \leqslant k)$ all lie in $G_{i}$.

We call a compact, orientable, irreducible, sufficiently large 3-manifold $M$ atoroidal if $M$ contains no essentially embedded annuli or tori.

2 ADDED IN PROOF. Each $T_{i}$ is homeomorphic to the residual space of a standard torus knot either in $S^{3}$ or in standard solid torus in $S^{3}$. 
DECOMPOSITION THEOREM. Let $(M, T)$ be a sufficiently-large 3-manifold pair. Then $M=N \cup \Sigma$ where (i) $\Sigma$ is the first term of a Seifert pair $(\Sigma, \Phi) \subset$ $(M, T)$ where the inclusion map of $(\Sigma, \Phi)$ into $(M, T)$ is essential, and $N$ is atoroidal; (ii) $N \cap \Sigma=\partial N \cap \partial \Sigma$ is a union of incompressible annuli and tori; (iii) if $(S, F)$ is a nondegenerate Seifert pair and $f:(S, F) \rightarrow(M, T)$ is an essential map, then $f$ can be deformed as a map of pairs to a map $g:(S, F) \rightarrow(M, T)$ with $g(S) \subset \Sigma, g(F) \subset T$; and (iv) no proper subcollection $\left(\Sigma^{\prime}, \Phi^{\prime}\right)$ of components of $(\Sigma, \Phi)$ satisfies (i)-(iii).

It is not difficult to show that the pair $(\Sigma, \Phi)$ satisfying the above is unique. We have conjectured that if $N$ is an atoroidal, sufficiently large 3-manifold, then $\pi_{1}(N)$ completely determines $N$ up to homeomorphism. A positive solution to this has been announced in [1].

\section{REFERENCES}

1. K. Johannson, Equivalences d'homotopie des variétés de dimension 3, C. R. Acad. Sci. Paris Sér. A (1975), A1009-A1010.

2. F. Waldhausen, On the determination of some bounded 3-manifolds by their fundamental groups alone, Proc. Internat. Sy mpos. on Topology and Appl. (Herceg-Novi, Yugoslavia, 1968), Savez Društava Matematičara, Fizičara I Astronoma, Belgrade, 1969, pp. 331-332. MR 42 \#2416.

DEPARTMENT OF MATHEMATICS, RICE UNIVERSITY, HOUSTON, TEXAS 77001 